ESAIM: PROCEEDINGS AND SURVEYS, December 2014, Vol. 47, p. 116-129

F. Abergel, M. Aiguier, D. Challet, P.-H. Cournède, G. Faÿ, P. Lafitte, Editors

\title{
BRANCHING AND AGGREGATION IN SELF-REPRODUCING SYSTEMS
}

\author{
VITALY VOLPERT ${ }^{1}$
}

\begin{abstract}
Self-reproducing systems (SRS) represent ensemble of objects (or individuals) which can produce other objects similar to themselves. If they compete with each other for resources, then they can form aggregates (or clusters) instead of a uniform distribution. New aggregates can split from the previous ones. In terms of biological populations, the aggregates correspond to biological species, and emergence of new aggregates to the process of speciation. Other examples of SRS will also be discussed.
\end{abstract}

Résumé. Les systèmes auto-reproductibles représentent des ensembles d'objets (ou d'individus) qui peuvent produire des objets similaires à eux-même. S'ils sont en compétition pour les ressources, alors ils peuvent former des agrégats (ou clusters) à la place des ditributions uniformes. Les nouveaux agrégats peuvent se séparer de ceux qui existent déjà. Pour les populations biologiques, les agrégats correspondent aux espèces et l'émergences de nouveaux agregats au processus de spéciation. Nous allons discuter les mécanismes qui régissent ces processus et quelques autres exemples.

\section{SELF-REPRODUCING SYSTEMS}

It was indicated by many authors that speciation is a general property of the living matter (see [11], [19] and references therein). Emergence of biological species is the most well known example widely discussed after the publication of Darwin's book on the origin of species. However there are many other examples, and the underlying mechanisms of speciation in these processes can be similar. In this work we will study this question in a mathematical framework which will allow us to formulate more precisely conditions of speciation. We will consider self-reproducing systems (SRS) which we understand in the following sense. We consider an ensemble of objects which can produce new objects similar to themselves, using the surrounding medium (resources). It is important to stress that these systems are not closed. We will show that if the elements of the system compete for resources, then this competition together with reproduction and variations of offsprings in comparison with their parents can result in the processes of aggregation and branching.

In order to define SRS more precisely, let us introduce the density distribution $u(x, t)$ of these objects. The space variable $x$ will be specified below, $t$ is time. We suppose that the evolution of the density distribution is described by the reaction-diffusion equation

$$
\frac{\partial u}{\partial t}=d \frac{\partial^{2} u}{\partial x^{2}}+W
$$

The diffusion term in this equation describes random motion (or random mutations), $W$ is the production rate which we will be mostly considered in the form

$$
W=a u^{k}(K-H(u))-\sigma u,
$$

\footnotetext{
${ }^{1}$ Institut Camille Jordan, UMR 5208 CNRS, University Lyon 1, 69622 Villeurbanne, France
}

(C) EDP Sciences, SMAI 2014 
where the first term in the right-hand side is the rate of natality and the second term the rate of mortality of the population. The natality rate is proportional to the population density in some power $k$ and to available resources. The rate of consumption of resources $H(u)$ can be proportional to the point-wise population density (local consumption), to the total population in some area (nonlocal consumption) or to the total population in the whole space (global consumption):

$$
H(u) \sim u, \quad \int_{\infty}^{\infty} \phi(x-y) u(y, t) d y, \quad \int_{-\infty}^{\infty} u(y, t) d y
$$

Nonlocal consumption of resources can be reduced to the local one if the kernel is the $\delta$-function, and to the global one if the kernel is identically constant. We will consider here various examples of SRS described by these models.

Biological populations. The most well-known example of SRS are biological populations, animals or cells. If $x$ is the physical space and $W=a u(K-b u)$, then we obtain the conventional logistic equation. In this case, the production rate $W$ is proportional to the population density and to available resources $(K-b u)$, which is the difference between the rate of renewing of resources $K$ and the rate of their consumption proportional to $u$.

Biological individuals can be characterized by their phenotype. For each phenotype $x$ and time $t$ we can consider the density distribution $u(x, t)$. Equation (1.1) implies in this case that the phenotype of offsprings is the same as the phenotype of parents plus a small random perturbation. It should be noted that the perturbation may not be small and the relation between phenotypes of parents and offsprings can be more complex.

In the case of sexual reproduction, we need to consider male and female subpopulations. Denote them by $v_{1}$ and $v_{2}$. Then instead of equation (1.1) we get the system of two equations

$$
\begin{aligned}
& \frac{\partial v_{1}}{\partial t}=d \frac{\partial^{2} v_{1}}{\partial x^{2}}+a v_{1} v_{2}\left(K-b\left(v_{1}+v_{2}\right)\right)-\sigma v_{1}, \\
& \frac{\partial v_{2}}{\partial t}=d \frac{\partial^{2} v_{2}}{\partial x^{2}}+a v_{1} v_{2}\left(K-b\left(v_{1}+v_{2}\right)\right)-\sigma v_{2} .
\end{aligned}
$$

We assume here that diffusion coefficients, the rate of reproduction and the mortality coefficient $\sigma$ are the same for males and females. Then the difference $z=v_{1}-v_{2}$ satisfies a linear parabolic equation. If $z(x, 0)=0$, that is the initial distributions of males and females are the same, then $z(x, t) \equiv 0$ for all times, and $v_{1}=v_{2}$. Hence we can reduce system $(1.2),(1.3)$ to the single equation with respect to the total population density $v=v_{1}+v_{2}$ :

$$
\frac{\partial v}{\partial t}=d \frac{\partial^{2} v}{\partial x^{2}}+\frac{a}{2} v^{2}(K-b v)-\sigma v
$$

This is a reaction-diffusion equation with a bistable nonlinearity. Asexual reproduction corresponds to the monostable nonlinearity. Properties of solutions are different in these two cases. We will discuss them in Section 2.

Economical populations. Another group of models describes distribution of wealth and manufactured objects. Suppose that a human population is uniformly distributed in space and the individuals in this population do not move. Each individual possesses some wealth. So the distribution of wealth $u(x, t)$ depends on the space variable $x$ and on time $t$. We will use the same equation (1.1) for the evolution of wealth distribution. The diffusion term describes local wealth redistribution from rich to poor where the flux is proportional to the wealth gradient. The rate of wealth production $W$ is determined by investments $I$ and resources $R$ :

$$
W=k I R-\sigma u \text {. }
$$

Here the second term in the right-hand side describes consumption of wealth. We suppose that investments is a function of wealth, $I=f(u)$, and $R=K-b u$, where $K$ is rate of renewal of resources and $b u$ is the rate of their consumption proportional to $u$. If we set $f(u)=a u^{k}$, then we get a logistic equation for $k=1$ and a bistable nonlinearity for $k=2$. 
Similar to biological populations, the space variable $x$ for economical populations can have a different interpretation. It is not the physical space with associate individuals, as in the previous example, but a space of manufactured objects. In some sense, this is similar to the space of phenotypes for biological populations. Manufactures objects can be characterized by certain number of properties (size, weight, material, and so on). This space of "phenotypes" is multi-dimensional. For simplicity, we will consider a one-dimensional space. We need to specify in what sense we understand self-reproduction of manufactured objects. When consumers buy some products, they create a demand and stimulate production of these objects. We suppose that the reproduction rate $W$ is proportional to the quantity of sold objects. In its turn, the latter is proportional to the quantity of objects $u$ and to the number of consumers which can buy these objects. This number of consumers equals the total number of consumers $K$ minus those who already bought these objects (proportional to $u$ ). Under these assumptions we get the logistic production rate: $W=a u(K-b u)$.

The diffusion term describes small variations in the properties of produced objects which we always observe in the market with several producers (e.g., computers and other electronic devices).

Intelligent populations. Similar to the economical populations, we consider here the physical space with individuals associated to each space point. The individuals do not move. Each of them possesses some knowledge. Instead of the distribution of wealth for economical populations, we consider now the distribution of knowledge $u(x, t)$ as a function of space and time. Knowledge is understood here in a wide sense and can involve any intellectual or spiritual activity (science, art, music, and so on). The rate of production of knowledge is proportional to the level of knowledge and to available resources. The latter, as before, is the difference between the rate $K$ of supply of resources and the rate of their consumption supposed to be proportional to $u$ (preservation of knowledge requires resources). Hence we obtain the same expression as before for the production rate, $W=a u(K-b u)$. The diffusion term in equation (1.1) describes local redistribution by means of resources necessary for knowledge production. Let us note that knowledge can diffuse through learning. In this case it goes from the place (individual) with greater knowledge to the place with lesser knowledge. It clearly increases the knowledge of the latter, however it does not directly decrease knowledge of the former as it should be according to the diffusion term. Indirect decrease of the knowledge production rate is caused by time spent for teaching.

If the space variable $x$ corresponds to the field of knowledge ("phenotype") and $u(x, t)$ is the number of people involved in this activity, then the reproduction consists in forming new specialists in this area. The reproduction rate $W$ is proportional to $u$ and to available resources, $W=u(K-b u)$. In the case of nonlocal consumption of resources, the process of speciation will lead to the emergence of an internal structure in the corresponding field of knowledge, as it is the case in science, literature or music.

Thus, different types of SRS are described by similar models. We will introduce in the next section nonlocal and global consumption of resources which describe intraspecific competition. We will show below that it determines two important properties of SRS, aggregation and branching.

\section{LOCAL, NONLOCAL AND GLOBAL CONSUMPTION OF RESOURCES}

Consider the reaction-diffusion equation

$$
\frac{\partial u}{\partial t}=d \frac{\partial^{2} u}{\partial x^{2}}+a u^{k}(1-H(u))-\sigma u .
$$

If $H(u)=u$, then we have local consumption of resources because they are consumed at the same space point $x$ where the individuals are located at this moment of time, where they reproduce and where they die. This equation is studied in detail both for $k=1$ (monostable case) and $k=2$ (bistable case) (see [33] and references therein).

Next, we will consider nonlocal consumption of resources where 


$$
H(u)=\int_{-\infty}^{\infty} \phi(x-y) u(y, t) d y,
$$

$\phi(x)$ is some kernel which shows how consumption of resources depends on distance: what is the rate of consumption of resources located at the point $x$ by the individuals located at the point $y$. Here we come to another concept where consumption of resources occurs in some area around the average location of the individuals. If $k=1$, equation (2.1) with nonlocal consumption of resources is the so-called nonlocal FisherKPP equation actively studied during the last decade (see [8], [21], [23], [24], [28]). The case $k=2$ was considered in $[7],[35]$.

In the case of global consumption of resources we set

$$
H(u)=s \int_{-\infty}^{\infty} u(y, t) d y .
$$

The integral here represents the total size of the population, $s$ is a constant. Hence consumption of resources occurs independently of the phenotype of the individuals. Reaction-diffusion equations with global resources were introduced in [35].

Reaction-diffusion equations with local and nonlocal consumption of resources describe propagation of waves, nonlocal consumption of resources describes speciation. Local and global consumption of resources describe pulse solutions. They are unstable in the case of local resources and they can become stable in the case of global resources. We will briefly recall the main results for the models of local consumption below in this section. Section 3 will be devoted to equations with nonlocal consumption of resources, and Section 4 to equations with global consumption.

In classical population dynamics, the population density $u(x, t)$ is described by the reaction-diffusion equation

$$
\frac{\partial u}{\partial t}=d \frac{\partial^{2} u}{\partial x^{2}}+F(u)
$$

where $F(u)=a u^{k}(1-u)-\sigma u$. It corresponds to equation $(2.1)$ with $H(u)=u$ (local consumption). If $k=1$, then $F(u)=0$ for two values of $u$ : $u_{+}=0$ and $u_{-}=1-\sigma / a$. If $\sigma<a$, then $u_{-}>0$, and there exist travelling wave solutions of equation $(2.2)$ with the limits $u_{ \pm}=u( \pm \infty)$. They were first studied in the context of population dynamics in [18], [26] and then in numerous other works (see [36] and references therein). These are solutions of the form $u(x, t)=w(x-c t)$, where $c$ is the wave speed. They satisfy the second-order ordinary differential equation

$$
d w^{\prime \prime}+c w^{\prime}+F(w)=0, \quad w( \pm \infty)=u_{ \pm}
$$

For all speeds $c$ greater than or equal to the minimal speed $c_{0}=2 \sqrt{d F^{\prime}(0)}$ these solutions are monotonically decreasing. They are stable in properly chosen spaces.

If $k=2$ and $\sigma / a<1 / 4$, then the function $F(u)$ has three zeros, $u_{+}=0$, and two others, $u^{*}$ and $u_{-}$, the roots of the equation $u(1-u)=\sigma / a, u_{+}<u^{*}<u_{-}$. There exists a solution of problem (2.3) for a unique value of speed $c$. The wave is monotone and globally asymptotically stable.

Equation (2.2) with $k=2$ has also a pulse solution, that is a stationary solution $w_{0}(x)$ with zero limits at infinity:

$$
d w_{0}^{\prime \prime}+F\left(w_{0}\right)=0, \quad w_{0}( \pm \infty)=0 .
$$

It can be easily constructed explicitly. This solution is unstable. Such solution does not exist for $k=1$. Let us note that the pulse solution exists for any positive values of parameters $a$ and $\sigma$. This means that the population persists even for high mortality rates. Moreover the maximum of the solution $w_{m}$ increases for greater $\sigma$ but the distribution becomes narrower. 


\section{Patterns And WAVES For nONLOCAL CONSUmption}

\subsection{Linear stability analysis}

Consider the reaction-diffusion equation with nonlocal consumption of resources in the monostable case:

Here

$$
\frac{\partial u}{\partial t}=d \frac{\partial^{2} u}{\partial x^{2}}+a u(1-J(u)), \quad x \in \mathbb{R} .
$$

$$
J(u)=\int_{-\infty}^{\infty} \phi(x-y) u(y, t) d y, \quad \int_{-\infty}^{\infty} \phi(y) d y=1 .
$$

Then $u=1$ is a stationary solution of this equation. Linearizing the equation about the stationary solution, we obtain the eigenvalue problem

$$
d v^{\prime \prime}-a J(v)=\lambda v .
$$

The Fourier transform gives the expression for the spectrum

$$
\lambda(\xi)=-d \xi^{2}-a \tilde{\phi}(\xi), \quad \xi \in \mathbb{R},
$$

where $\tilde{\phi}(\xi)$ is the Fourier transform of the function $\phi$. If we replace $\phi(x)$ by the $\delta$-function, then we return to the local reaction-diffusion equation, $\tilde{\phi}(\xi)=1$, and $\lambda(\xi)<0$ for all $\xi$. In the nonlocal case, if $\tilde{\phi}(\xi)$ is negative for some $\xi$, then $\lambda(\xi)$ can become positive, and the uniform solution $u=1$ can become unstable. If we set

then

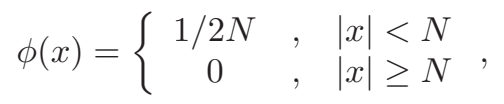

$$
\lambda(\xi)=-d \xi^{2}-a \sin (\xi N) /(\xi N) .
$$

If we consider a bounded interval with certain boundary conditions (Dirichlet, Neumann, periodic), then the spectrum is discrete, and the conditions of the existence of positive eigenvalues can be expressed through the parameters $d, a, N$.

\subsection{Bifurcations}

If a simple real eigenvalue crosses the origin, then the homogeneous in space solution loses its stability and a nonhomogeneous solution bifurcates from it. Bifurcation analysis for the nonlocal reaction-diffusion equation was first carried out in [8] (see also [35]). We will not stop here on the detail of this analysis and will restrict ourselves to the numerical results shown in Figure 1.

In the case of the piece-wise constant function $\phi(x)$, the spectrum is given by formula (3.2). For $d$ sufficiently large, it lies in the left-half plane and the homogeneous in space solution $u=1$ is stable. When we decrease $d$, a real eigenvalue crosses the origin resulting in bifurcation of a periodic in space solution (Figure 1 (left)). Let us note that the total mass of the stationary solution $u(x)$, that is the expression

$$
M(u)=\frac{1}{L} \int_{0}^{L} u(x) d x
$$

grows as $d$ decreases. Figure 1 (right) shows its difference with the total mass of the homogeneous solutions $M_{0}=1$. This conclusion is important for the applications. We will return to them in the discussion section below. It is interesting to note that $M(u)$ does not have a square root behavior near the bifurcation point. Setting

$$
u=u_{0}+\epsilon u_{1}(x)+\epsilon^{2} u_{2}(x)
$$



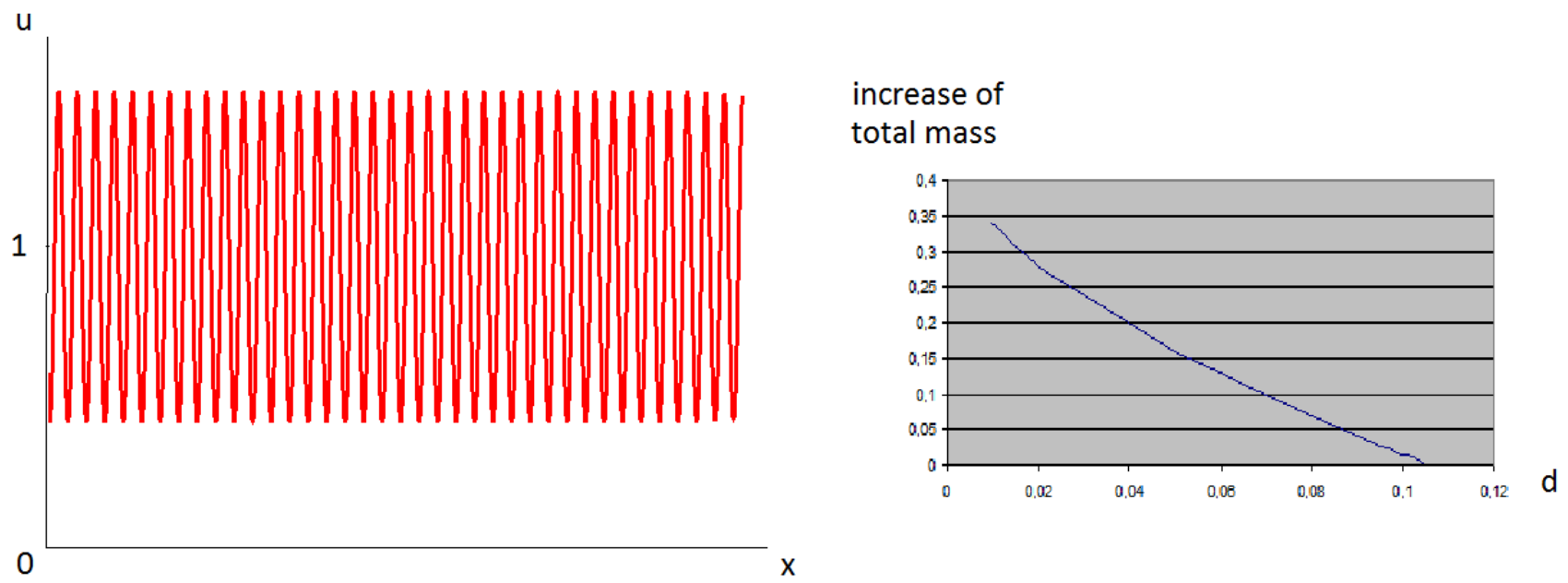

Figure 1. Periodic solution which bifurcates from the homogeneous solution (left). The integral of the periodic solution increases when the diffusion coefficient decreases (right).

in the bifurcation analysis, where $u_{0}=1, \epsilon=\sqrt{d_{c}-d}$ and $d_{c}$ is the critical value of the diffusion coefficient, we note that the amplitude of the periodic solution grows as a square root. However, $\operatorname{since} u_{1}(x)=\sin (k x)$ for some value of $k$, then this term vanishes in $M(u)$. Only the next term, linear with respect to the difference $d_{c}-d$ is preserved after integration.

\subsection{Periodic travelling waves and branching}

If we now consider a localized initial condition with a finite support, then we can expect propagation of a travelling wave similar to the local reaction-diffusion equation. However, as we discussed above, the homogeneous in space solution $u=u_{0}$ behind the wave can become unstable. In order to explain behavior of solutions in this case, we need to consider two travelling waves. One of them between constant solutions $u=0$ and $u=u_{0}$. We will call it $\left[0, u_{0}\right]$-wave. Another one is a wave between unstable homogeneous solution $u_{0}$ and a periodic in space structure $u_{p}(x)$. It is a generalized travelling wave which we will call $\left[u_{0}, u_{p}\right]$-wave. Let $c_{0}$ be the speed of the former and $c_{p}$ of the latter. If $c_{0}>c_{p}$, then the distance between them grows, and we observe a stable (on the half-axis) propagation of the $\left[0, u_{0}\right]$-wave with an unstable equilibrium $u_{0}$ behind it. If $c_{0}<c_{p}$, then the generalized travelling wave moves faster, they merge and form a single periodic wave. Both types of behavior are observed numerically [37]. Such behavior is well known in the theory of travelling waves (wave trains, systems of waves, decomposition of waves) [33] with the difference that the equilibrium separating two waves is usually stable.

Figure 2 shows a numerical example where the two waves merge and form a single periodic wave. A stationary periodic structure is formed behind the wave (left). The maxima of the solution at the $(x, t)$-plane form specific branching pattern (right). It has an important interpretation from the point of view of speciation. It was observed before already in numerous works [20]- [22], [24], [31]. We will return to this question below.

\section{4. $2 \mathrm{D}$ equation}

In the two-dimensional space, behavior of solutions of the equation

where

$$
\frac{\partial u}{\partial t}=d \Delta u+a u(1-J(u))
$$



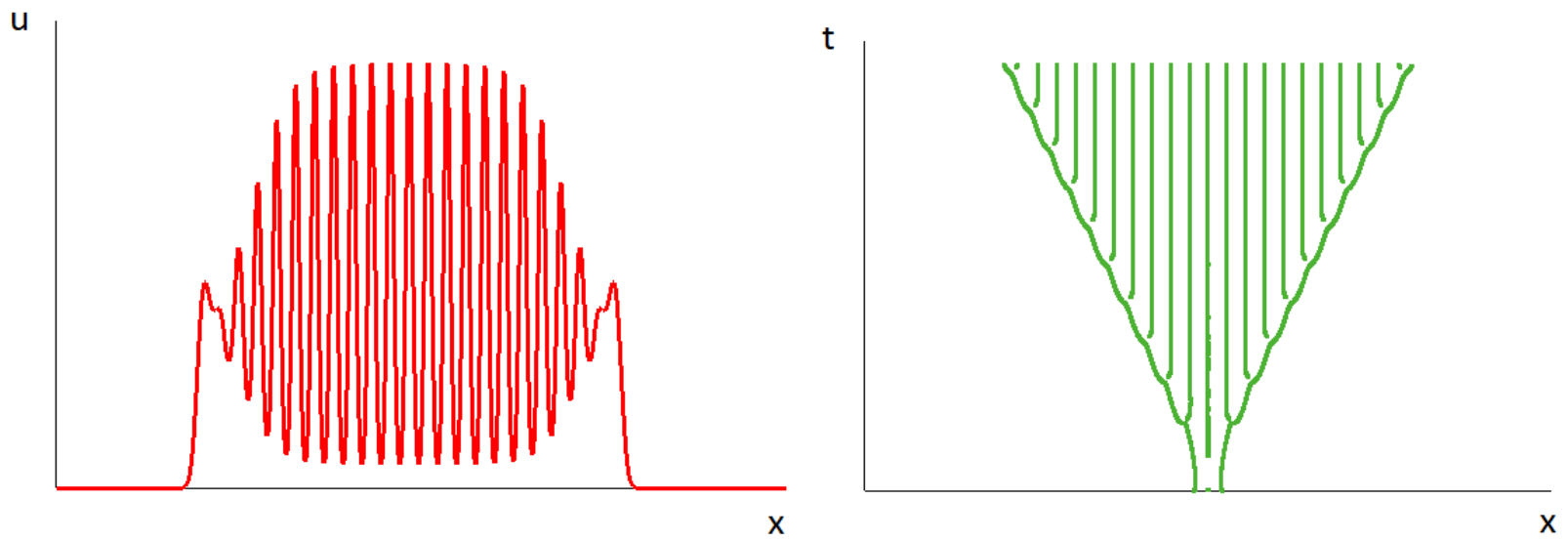

FIgURE 2. Propagation of a periodic wave in the monostable case with nonlocal consumption or resources (equation (3.1)) for $a=5, s=0.2, d=0.3$. Snapshot of solution (left), coordinates of the maxima of the solution on the $(x, t)$-plane (right).

$$
J(u)=\int_{\mathbb{R}^{2}} \phi\left(x_{1}-y_{1}, x_{2}-y_{2}\right) u\left(y_{1}, y_{2}\right) d y_{1} d y_{2}
$$

depends on the support of the function $\phi$.
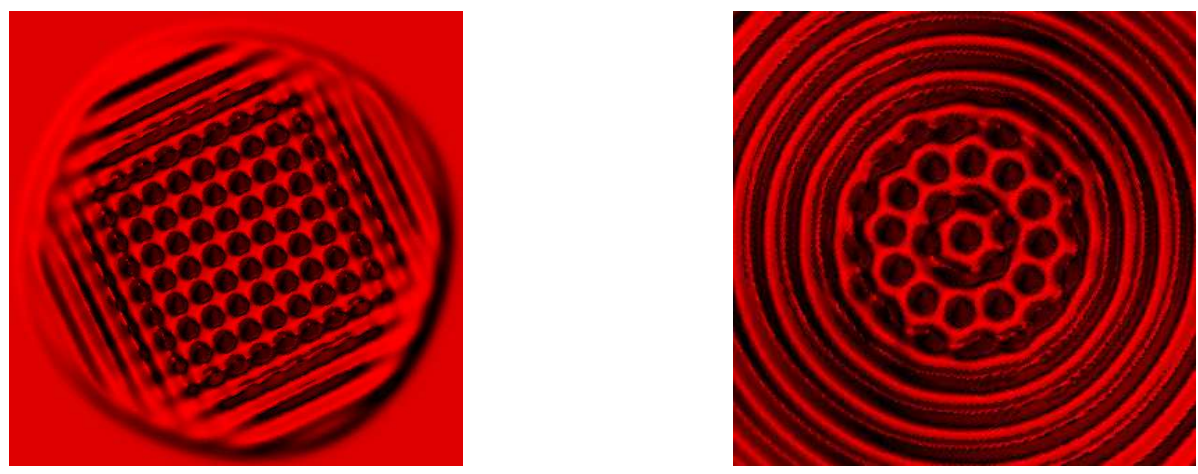

Figure 3. Propagation of circular wave and formation of a structure behind the wave. The maxima of the density form a square grid in the case of a square support of $\phi$ (left) and a circular structure in the case of a circular support (right). Reprinted with permission from [37].

Two examples are shown in Figure 3 where the piece-wise constant function $\phi$ has a square support (left) or a circular support (right). In both cases, there is a circular wave propagating outwards. However the pattern behind the wave depends on the support of the function $\phi$.

We discussed in this section nonlocal reaction-diffusion equation in the monostable case. The bistable case is qualitatively similar in the sense that the homogeneous in space solution can lose its stability resulting in appearance of stationary nonhomogeneous solutions. 


\section{Simple AND MUltiple PUlSes}

\subsection{Existence and stability of pulses}

Let us consider the reaction-diffusion equation in the bistable case

with global consumption of resources,

$$
\frac{\partial u}{\partial t}=d \frac{\partial^{2} u}{\partial x^{2}}+a u^{2}(1-s I(u))-b u
$$

$$
I(u)=\int_{-\infty}^{\infty} u(y, t) d y .
$$

Here $a, b$ and $s$ are positive numbers. Existence of stationary solutions of this equation can be easily studied. The equation

$$
w^{\prime \prime}+a w^{2}(1-s I(w))-b w=0, \quad w( \pm \infty)=0
$$

can be solved analytically. It can be verified that there exist two solutions (up to translation in space) if $0<s<s_{0}$ for some critical value $s_{0}$, and there are no solutions for $s>s_{0}$ [35]. Hence existence of solutions is determined by the level of global consumption of resources. Let us note that such solutions do not exist in the monostable case (asexual reproduction).

Denote a stationary solution of problem (4.2) by $w_{0}$ and consider the eigenvalue problem for the equation linearized about this solution:

$$
L v \equiv v^{\prime \prime}+2 a c v-b v-a s w_{0}^{2} I(v)=\lambda v, \quad w( \pm \infty)=0,
$$

where $c=1-s I\left(w_{0}\right)$. The operator

$$
L_{0} v=v^{\prime \prime}+2 a c v-b v
$$

has a positive eigenvalue. Indeed, the function $w_{0}^{\prime}(x)$ is the eigenfunction of this operator corresponding to the zero eigenvalue. It is related to the invariance of solutions with respect to translation. Since it has variable sign, it cannot be the principal eigenfunction, that is the eigenfunction corresponding to the eigenvalue with the maximal real part. It is known that such eigenvalue is real, positive and the corresponding eigenfunction is positive (up to a constant factor) [33]. Therefore $\lambda=0$ is not the principal eigenvalue of the operator $L_{0}$, and it has a positive eigenvalue. Hence the pulse solution $w_{0}(x)$ is an unstable stationary solution of the local reaction-diffusion equation.

Numerical simulations show that this solution can be a stable stationary solution of equation (4.1). This means that global consumption of resources can stabilize it. The spectrum of problem (4.3) is not yet studied analytically. Some model examples show that the integral term can remove the positive eigenvalue [35].

\subsection{Multiple pulses}

In this section we consider equation (1.1) where

$$
W=a u^{k}\left(1-s_{1} J(u)\right)\left(1-s_{2} I(u)\right)-\sigma u .
$$

It describes dynamics of the population with two resources, one of them is nonlocal and another one is global in the sense of the previous sections. Both resources are supposed to be necessary for reproduction, so that the reproduction rate is proportional to the product of the two factors. If we put one of the constants $s_{1}$ or $s_{2}$ equal to zero, then we get one of the previous models.

The presence of the integral $J(u)$ provides splitting of the population into sub-populations (speciation) while the integral $I(u)$ in the reproduction term limits the total size of the population. As a result, instead of travelling waves and single pulses observed before we obtain here solutions which consists of multiple pulses (Figure 5). 


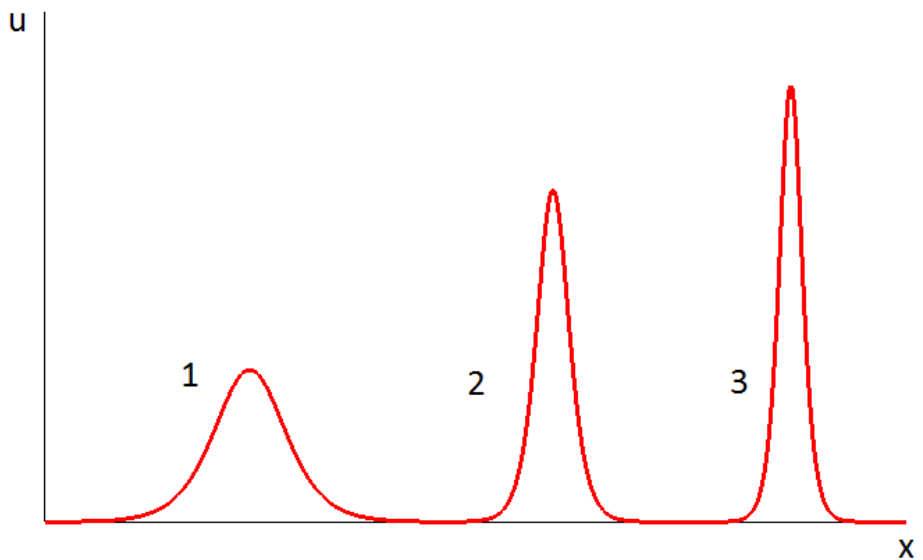

FIGURE 4. Srationary solutions of equation (4.1) for $a=1, s=0.1, d=0.03,1 . \sigma=0.001,2$. $\sigma=0.005,3 . \sigma=0.01$.
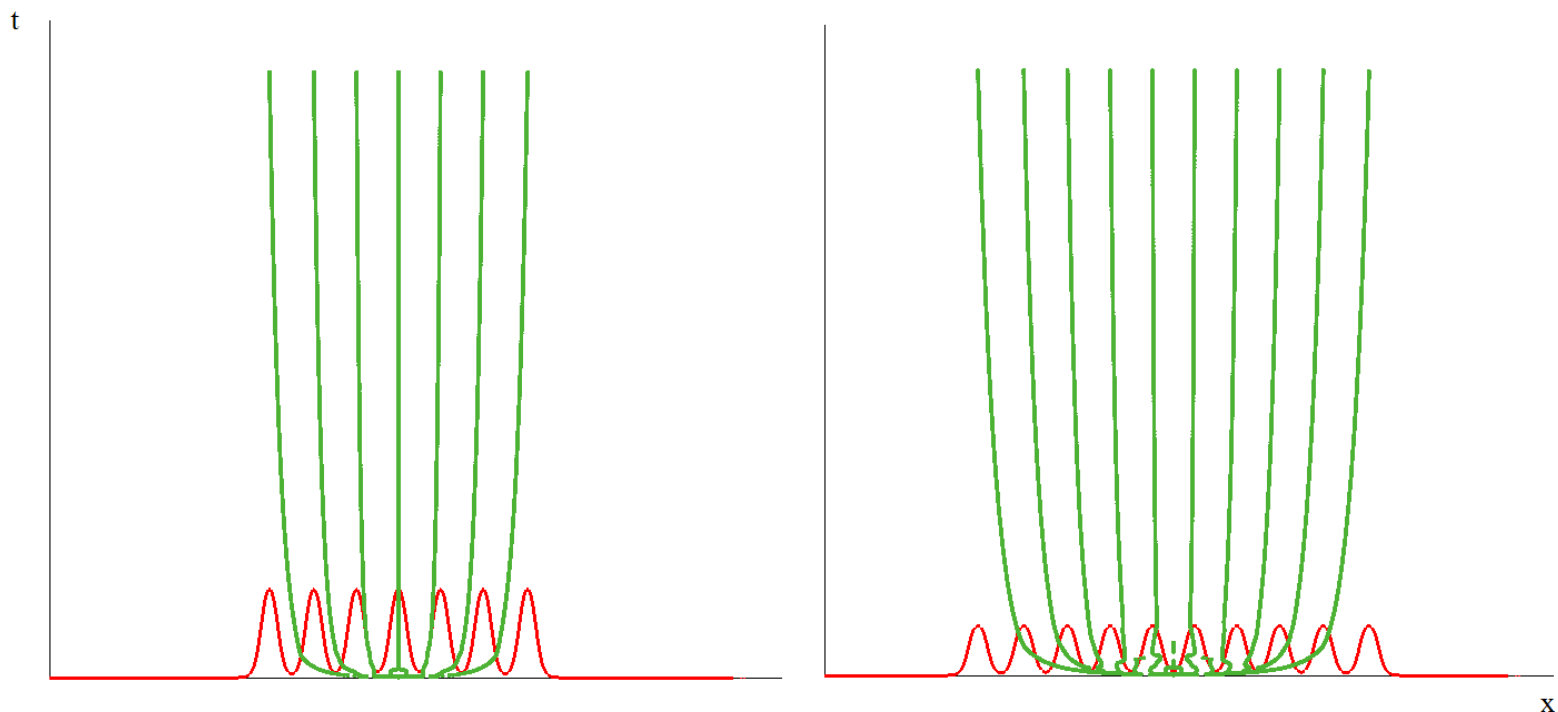

FIGURE 5. Seven and ten pulse solutions obtained respectively for $s_{2}=0.15 ; 0.1$ and the values of other parameters $k=2, D=0.1, N=3, a=1, b_{1}=2, b_{2}=10, \sigma=0.1, s_{1}=1$. The red curves are the function $u(x, t)$ at a given moment of time, the green curves show the positions of the maxima of the solution in the $(x, t)$-plane.

Their number depends on the value of the parameter $s_{2}$. If we decrease it, the number of pulses increases. Let us also note that the distance between pulses slowly grows in time. So multiple pulse solutions are not stationary but transient solutions. When the distance between pulses tends to infinity, each of them converges to the stationary single pulse solution. 


\section{Discussion}

\subsection{General properties of self-reproducing systems}

Aggregation and branching are common properties of self-reproducing systems that may not depend on their specific origin.

Aggregation. The objects (individuals) that form self-reproducing systems can be either uniformly distributed in space or show spatial organization. In the case of local consumption of resources described by local scalar reaction-diffusion equations only uniform distributions are stable. In the case of nonlocal consumption of resources, which describes competition for resources, nonuniform density distributions can become stable.

There are two ways to obtain stable nonuniform distributions: due to bifurcations from a uniform solution or as a pulse solution. Homogeneous in space solutions can lose their stability in the case of nonlocal consumption of resources resulting in the emergence of nonhomogeneous solutions. If the bifurcation parameter (e.g., diffusion coefficient) moves further in the instability region, the density distribution becomes strongly inhomogeneous with localized regions of high density, which we call here aggregates or clusters. Such behavior is observed for both sexual and asexual reproduction in the case of nonlocal consumption of resources.

Stable stationary pulses are observed in the bistable case (sexual reproduction) with global consumption of resources. In the monostable case or in the case of nonlocal consumption of resources such solutions were not observed. We can conjecture that they do not exist. In the bistable case with local consumption of resources such solutions exist but they are unstable.

These are the simplest patterns observed for nonlocal and global consumption of resources. More complex models can produce other patterns. For example, multiple pulses in the case of two resources, nonlocal and global.

Branching (speciation). Clusters or aggregates formed due to the nonuniform density distribution can split resulting in appearance of new clusters. In the case of biological species this process is called speciation. It describes the emergence of biological species in the process of evolution. It is widely studied with various modelling approaches [11], [19]. Nonlocal reaction-diffusion equations were used to describe speciation in [20][22], [24], [31] (Section 3.3).

The minimal model to describe speciation takes into account the following three properties: nonlocal consumption of resources related to the intraspecific competition, reproduction with the same phenotype, small perturbations of the phenotype due to random mutations. A similar mechanism of speciation can be identified in numerous other models of biological, economical and intelligent populations.

Species. The notion of biological species is largely discussed in literature, and we will not discuss it here. From the point of view of the modelling presented in this work, we can interpret species as localized structures (aggregates) in the space of phenotypes. Such localized structures exist due to nonlocal or global consumption of resources and, as a consequence, competition for resources. This approach can be used to model emergence and evolution of biological species (previous paragraph). In particular, introducing appropriate models with nonlocal and global consumption of resources we can describe all main patterns of Darwin's diagram [7].

\subsection{Two more examples}

We will present here two other examples of self-reproducing systems where the process of aggregation has interesting biological applications.

Ants and specialization of workers. Let the space variable $x$ correspond to various tasks that should be accomplished and $u(x, t)$ to the number of workers involved in this process. In the case of ants, this specialization is also related to their phenotype, in the case of workers to their training. Denote by $K$ the amount of work which should be done for each task during given time interval, and $b u$ is the work already accomplished (proportional to the number of workers). Then we can use equation (1.1) for the distribution of $u$. The diffusion term shows that phenotype of ants can change due to small variations. The production term $W$ is proportional to the remaining amount of work, $W=a(K-b u)$. 
If we take into account that workers (ants) can fulfil various tasks in some range of their competence, then we obtain the nonlocal term $W=a(K-b J(u))$. In this case we obtain a linear equation with a homogeneous in space stationary solution which can become unstable. The drawback of this equation is that its solution is not uniformly bounded and can become negative.

Next, let us take into account that the rate of production of workers can depend on their number. In the case of ants, the workers can reproduce themselves [9]. It can be a linear dependence on their number or a nonlinear dependence if their interaction increases the efficiency of the reproduction. Then we get $W=a u^{k}(K-b J(u))$ where $k$ is a positive number. Together with the term which describes retirement of workers (mortality of ants) we arrive to the equation

$$
\frac{\partial u}{\partial t}=d \frac{\partial^{2} u}{\partial x^{2}}+a u^{k}(K-b J(u))-\sigma u .
$$

Finally if the global number of workers (ants) is limited because of limited resources, then the production rate depends also on global consumption of resources:

$$
\frac{\partial u}{\partial t}=d \frac{\partial^{2} u}{\partial x^{2}}+a u^{k}\left(K_{1}-b_{1} J(u)\right)\left(K_{2}-b_{2} I(u)\right)-\sigma u .
$$

The model of specialization of workers is based on two types of signaling. The first one controls the work to be done, the second one from the workers with the purpose to increase their number. This model is considered in Section 4.2. Multiple pulses observed there correspond to the groups of specialized workers.

Density dependent proliferation. Cell proliferation can depend on the average cell density. There are various mechanisms which can be involved: mechanical pressure, various signals coming from the surrounding cells or nutrients and hormones coming from outside and consumed by cells. Let us introduce the average cell density

$$
\bar{u}(x)=\frac{1}{2 N} \int_{x_{-} N}^{x+N} u(y) d y
$$

and consider the equation

Note that

$$
\frac{\partial u}{\partial t}=d \frac{\partial^{2} u}{\partial x^{2}}+a u^{k}(K-b \bar{u})-\sigma u
$$

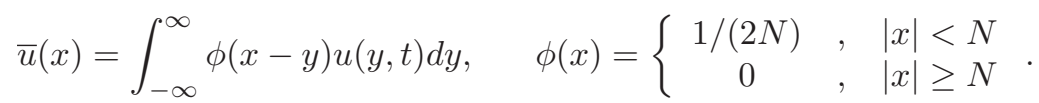

So equation (5.6) is a particular form of nonlocal reaction-diffusion equations considered above. Instability and branching described by the nonlocal reaction-diffusion equation is related to branching patterns in angiogenesis and in plants.

\subsection{Models of reproduction}

The reproduction terms in the models considered above are simplified. We will briefly discuss here more complete models. We should also note that biological justification of the models of sexual reproduction is quite complex and it requires further investigation.

Phenotypes of mating partners and offsprings. Average phenotypes of mating partners are generally not the same but they are related to each other [27]. If the space variable $x$ corresponds to the phenotype, then the reproduction terms become proportional to the densities of males $v_{1}(x)$ and females $v_{2}(x+h)$ taken at different space points. Hence we consider shifted phenotypes. Solutions of the corresponding equations are also shifted.

Next, we need to take into account phenotype distributions around some average values. Moreover, phenotypes of offsprings can be different from the phenotypes of parents. In this case, there are additional nonlocal 
terms in the model, and the reproduction terms become more complex ( [35], page 537). These models should be studied in more detail.

Human height distribution. Let us discuss the rate of sexual reproduction taking as example the human height distribution. In this case, the space variable $x$ is height. Consider the woman height distribution $u(x, t)$ described by the equation

$$
\frac{\partial u}{\partial t}=d \frac{\partial^{2} u}{\partial x^{2}}+(B-D) u
$$

where $B$ is the birth and $D$ is the death rates. Looking for a solution of this equation in the form $u(x, t)=$ $e^{\lambda t} v(x),(\lambda \geq 0)$ we get

$$
d v^{\prime \prime}+(B-D-\lambda) v=0 .
$$

The distribution $v(x)$ has a specific bell shape (Figure 6). It implies that $v^{\prime \prime}(x)<0$ in some interval of heights $x_{1}<x<x_{2}\left(x_{1} \approx 155 \mathrm{~cm}, x_{2} \approx 165 \mathrm{~cm}\right)$ and $v^{\prime \prime}(x)>0$ outside this interval. Hence $B>D-\lambda$ inside the interval and the inequality is opposite outside it. Setting $B=F w$, where $F$ is fertility and $w$ is the proportion of married women ${ }^{1}$, and assuming that $F$ and $D$ are constant in this interval of heights [10], we conclude that $w$ depends on $x$. This conclusion is in agreement with statistical data that the probability of marriage and having children depends on height [10], [30], [32]. Even a weak dependence of the proportion of married women on height can change the sign of the coefficient $B-D-\lambda$, which is necessary for the existence of the bell shape distributions.

A plausible explanation of height dependent marriage probability for women is related to the number of available men. We recall that heights of men and women forming a couple are related to each other [27]. Hence $w(x) \sim z(x+h)$, where $z(x)$ is the man height distribution, $h$ is some number. Therefore the reproduction rate is proportional to the product of man and woman densities. System (1.2), (1.3) with global consumption of resources and with a shift of heights gives a good approximation of human height distribution (Figure 6).

Reproduction and mortality rates depend on the phenotype. Biological species correspond to localized solutions in the space of phenotypes. As we discussed above, in the bistable case with the global consumption of resources such solutions exist and they are stable. If the rates of reproduction or mortality depend on the phenotype, then this localized solution will move in the direction where the reproduction increases or mortality decreases [7], [35].

The models with space dependent coefficients are used in modelling of natural selection [7]. If we assume, according to Darwin, that the most adapted survive and multiply while less adapted disappear, then we need to assume that survival and reproduction depend on the phenotype, that is on the space variable. This assumption allows us to describe the process of natural selection.

In the bistable case, localized solution are influenced by space dependent coefficients but they exist also for constant coefficients. This is different in the monostable case where localized solution can exist if the reproduction rate is greater than the mortality rate only inside some finite intervals of phenotypes (or genotypes). This is the case in cell population dynamics where these existence intervals correspond to different cell types.

\section{ACKNOWLEDGEMENTS}

The author is grateful to the referees for the stimulating discussion. Numerical simulations shown in Figure 6 are carried out by N. Reinberg.

\footnotetext{
${ }^{1}$ We associate here for simplicity marriage and having children.
} 


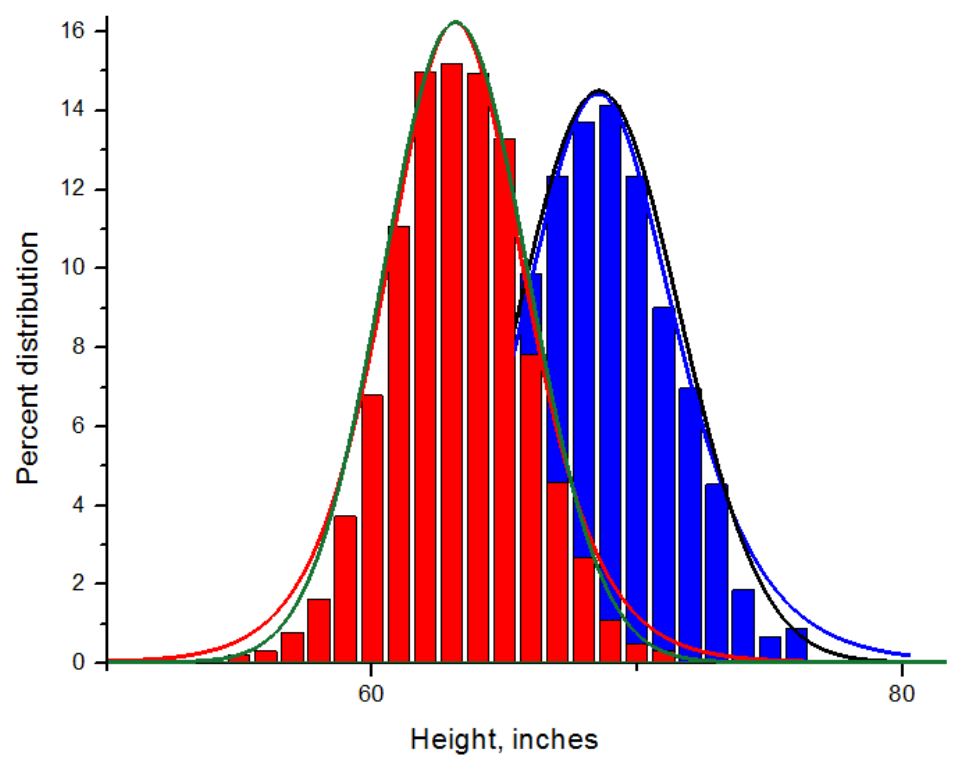

FIgURE 6. Human height distribution in statistical data (histograms) [1], numerical simulations with the nonlocal reaction-diffusion system (upper curves) and normal distributions (lower curves). Women height distribution is shown in red, men distribution in blue.

\section{REFERENCES}

[1] S. Abraham. Weight and height of adults 18-74 years of age, United States, 1971-74. Vital and health statistics: Series 11, Data from the National Health Survey; no. 211. DHEW publication; (PHS), 79-1659.

[2] N. Apreutesei, N. Bessonov, V. Volpert, V. Vougalter. Spatial structures and generalized travelling waves for an integrodifferential equation. DCDS B, 13 (2010), no. 3, 537-557.

[3] N. Apreutesei, A. Ducrot, V. Volpert. Travelling waves for integro-differential equations in population dynamics. Discrete Contin. Dyn. Syst., Ser. B, 11 (2009), no. 3, 541-561.

[4] N. Apreutesei, A. Ducrot, V. Volpert. Competition of species with intra-specific competition. Math. Model. Nat. Phenom., 3 (2008), 1-27.

[5] S. Atamas. Self-organization in computer simulated selective systems. Biosystems, 39 (1996), 143-151.

[6] H. Berestycki, G. Nadin, B. Perthame, L. Ryzhik. The non-local Fisher-KPP equation: travelling waves and steady states. Nonlinearity, 22 (2009), no. 12, 2813-2844.

[7] N. Bessonov, N. Reinberg, V. Volpert. Mathematics of Darwin's diagram. Math. Model. Nat. Phenom., 9 (2014), in press.

[8] N.F. Britton. Spatial structures and periodic travelling waves in an integro-differential reaction-diffusion population model. SIAM J. Appl. Math., 6 (1990), 1663-1688.

[9] E. Brunner, J. Kroiss, A. Trindl, J. Heinze. Queen pheromones in Temnothorax ants: control or honest signal? BMC Evolutionary Biology 2011, 11:55.

[10] P. Cahu, F. Fall, R. Pongou. Demographic transition in Africa: the polygamy and fertility nexus, 2011, http://congres.afse.fr/docs/2011/410307demographictransitionpolygamy.pdf

[11] J.A. Coyne, H.A. Orr. Speciation. Sinauer Associates, Sunderland, 2004.

[12] C. Darwin. The origin of species by means of natural selection. Barnes \& Noble Books, New York, 2004. Publication prepared on the basis of the first edition appeared in 1859 .

[13] I. Demin, V. Volpert. Existence of waves for a nonlocal reaction-diffusion equation. Math. Model. Nat. Phenom., 5 (2010), no. $5,80-101$.

[14] L. Desvillettes, P.E. Jabin, S. Mischler, G. Raoul. On selection dynamics for continuous structured populations. Commun. Math. Sci., 6 (2008), no. 3, 729-747.

[15] U. Dieckmann, M. Doebeli. On the origin of species by sympatric speciation. Nature, 400 (1999), 354-357.

[16] M. Doebeli, U. Dieckmann. Evolutionary branching and sympatric speciation caused by different types of ecological interactions. The American Naturalist, 156 (2000), S77-S101. 
[17] A. Ducrot, M. Marion, V. Volpert. Spectrum of some integro-differential operators and stability of travelling waves. Nonlinear Analysis Series A: Theory, Methods and Applications, 74 (2011), no. 13, 4455-4473.

[18] R.A. Fisher. The wave of advance of advantageous genes. Ann. Eugenics, 7 (1937), 355-369.

[19] S. Gavrilets. Fitness Landscape and the Origin of Species. Princeton University Press, Princeton, 2004.

[20] S. Genieys, N. Bessonov, V. Volpert. Mathematical model of evolutionary branching. Mathematical and computer modelling, 2008, doi: 10/1016/j.mcm.2008.07.023

[21] S. Genieys, V. Volpert, P. Auger. Pattern and waves for a model in population dynamics with nonlocal consumption of resources. Math. Model. Nat. Phenom., 1 (2006), no. 1, 65-82.

[22] S. Genieys, V. Volpert, P. Auger. Adaptive dynamics: modelling Darwin's divergence principle. Comptes Rendus Biologies, 329 (2006) no. 11, 876-879.

[23] S.A. Gourley. Travelling front solutions of a nonlocal Fisher equation, J. Math. Biol., 41 (2000), 272-284.

[24] S.A. Gourley, M.A.J. Chaplain, F.A. Davidson. Spatio-temporal pattern formation in a nonlocal reaction-diffusion equation. Dynamical systems, 16 (2001), no. 2, 173-192.

[25] D. Iron, M.J. Ward. A metastable spike solution for a nonlocal reaction-diffusion model. SIAM J. Appl. Math., 60 (2000), no. 3, $778-802$.

[26] A.N. Kolmogorov, I.G. Petrovsky, N.S. Piskunov. A study of the diffusion equation with increase in the amount of substance, and its application to a biological problem. Bull. Moscow Univ., Math. Mech., 1:6 (1937), 1-26. In: Selected Works of A.N. Kolmogorov, Vol. 1, V.M. Tikhomirov, Editor, Kluwer, London, 1991

[27] I.C. McManus, C.G.N. Mascie-Taylor. Human assortative mating for height: non-linearity and heteroscedasticity. Human Biology, 56 (1984), no. 4, 617-623.

[28] G. Nadin, L. Rossi, L. Ryzhik, B. Perthame. Wave-like solutions for nonlocal reaction-diffusion equations: a toy model. Math. Model. Nat.Phenom., 8 (2013), no. 3, 33-41.

[29] Y. Nec, M. J. Ward. The stability and slow dynamics of two-spike patterns for a class of reaction-diffusion system. Math. Model. Nat. Phenom., 8 (2013), no.5.

[30] B. Pawlowski, R.I.M. Dunbar, A. Lipowicz. Tall men have more reproductive success. Nature, 403 (2000), 156.

[31] B. Perthame, S. Genieys. Concentration in the nonlocal Fisher equation: the Hamilton-Jacobi limit. Math. Model. Nat. Phenom., 4 (2007), 135-151.

[32] R. Sear. Size-dependent reproductive success in Gambian men : does height or weight matter more ? Biodemography and Social Biology, 53 (2006), no. 3-4, 172-188.

[33] A.I. Volpert, V. Volpert, V.A. Volpert. Traveling wave solutions of parabolic systems. Translation of Mathematical Monographs, Vol. 140, AMS, Providence, 1994.

[34] V. Volpert. Elliptic partial differential equations. Volume 1. Fredholm theory of elliptic problems in unbounded domains. Birkhäuser, 2011.

[35] V. Volpert. Elliptic partial differential equations. Volume 2. Reaction-diffusion equations. Birkhäuser, 2014.

[36] V. Volpert, S. Petrovskii. Reaction-diffusion waves in biology. Physics of Life Reviews, 6 (2009), 267-310.

[37] V. Volpert, V. Vougalter. Emergence and propagation of patterns in nonlocal reaction-diffusion equations arising in the theory of speciation. In: Dispersal, individual movement and spatial ecology. M. Lewis, Ph. Maini, S. Petrovskii. Editors. Springer Applied Interdisciplinary Mathematics Series, in press. 